\title{
Influence of Landscape Design on Driving Behavior Based on Slope Illusion
}

\author{
Hao Li and Yueyang Zhang (iD) \\ Architecture and Civil Engineering, Hubei University of Technology, 430070 Wuhan, China \\ Correspondence should be addressed to Yueyang Zhang; 1536476721@qq.com
}

Received 22 October 2021; Accepted 31 December 2021; Published 12 January 2022

Academic Editor: Filomena Mauriello

Copyright (c) $2022 \mathrm{Hao} \mathrm{Li}$ and Yueyang Zhang. This is an open access article distributed under the Creative Commons Attribution License, which permits unrestricted use, distribution, and reproduction in any medium, provided the original work is properly cited.

\begin{abstract}
In a continuous downhill section of a mountain highway, factors such as road alignment, roadside environment, and other visual characteristics will impact the slope illusion drivers experience and engage in unsafe driving behaviors. To improve the negative consequences of slope illusion and driving safety in continuous downhill sections, the effects of plant spacing, height, roadside distance, and color on driving behavior were all studied by simulating the plant landscape in a virtual environment. A driving simulator and UC-win/road software were used to conduct an indoor driving simulation experiment, and parameters such as speed and lateral position offset were used as the evaluation indices of driving stability to reflect the driver's speed perception ability with subjective equivalent speeds. The results show that a plant landscape with appropriate plant spacing, height, roadside separation, and color is conducive to improving driving stability. Furthermore, a landscape with a height of $3 \mathrm{~m}$, spacing of $10 \mathrm{~m}$, roadside spacing of $0.75 \mathrm{~m}$, and appropriate color matching can enhance the slope perception ability and speed perception ability of drivers, which is conducive to improving the driving safety of continuous downhill sections.
\end{abstract}

\section{Introduction}

Drivers obtain traffic information through vision, which plays a key role in the vehicle operation when driving. However, due to the influence of personal, environmental, and road alignment factors, a driver's visual perception will deviate, especially when driving on continuous downhill sections. On these sections, drivers often develop an illusion of the true slope of the road due to the change of slope over distances. This slope illusion can cause the driver to misjudge the state of the road, resulting in an incorrect evaluation of driving speed, and increase the potential of traffic accidents.

To avoid the adverse effects of visual misjudgment, scholars have studied the mechanism and treatment of visual illusions. In "illusion and its application in highway alignment design," Yuan and Cheng [1] proposed that the influence of illusion on drivers should be considered in road design and proposed for the first time to correct the visual illusion of road by using the principle of perspective view. Based on the visual characteristics of drivers, $\mathrm{Lu}$ [2] suggested controlling the visual deviation of road sections by optimizing the linear combination. Guo et al. [3] analyzed the causes of slope illusion and its impact on driving safety based on animation simulation analysis. Qi [4] analyzed the formation mechanism of slope illusions from the following three aspects: road alignment, roadside environment, and driver visual characteristics, and concluded that the slope illusion can be corrected by setting plant landscape, adding inclined guardrails, and widening the road.

The road landscape can not only provide accurate line of sight guidance for drivers but also keep drivers in a good psychological state during driving. Additionally, the roadside landscape provides a speed reference for drivers, which is conducive to drivers' speed control. Therefore, taking a continuous long downhill as the research object, the plant landscape can be set to improve the slope illusion during downhill driving and to explore the influence of plant landscape factors on driving behavior. The purpose is to 
improve the driving safety of continuous downhill sections by designing plant landscapes.

\subsection{Formation Mechanism and Treatment Measures of Slope} Illusion. The following two factors are used for the generation of slope illusion: the driver's own physiological and psychological aspects and the slope length and difference between the front and rear slopes during downhill driving. A driver is most likely to have slope illusion due to the influence of slope length and front and rear slope differences. The longer the front slope and the greater the front and rear slope difference are, the more likely a slope illusion will occur. Relevant research shows that the slope difference that may produce a slope illusion has a certain critical value. When the front and rear slope difference of a road section is greater than $2.0 \%$, the driver may have a slope illusion when driving on this road section. When the current rear slope difference exceeds $2.5 \%$, the slope illusion is more obvious [2]. When the road slope changes, a driver's perception of the slope change can only last for approximately $10 \mathrm{~s}$, and then it will adapt to the slope. When a driver stays on the front slope for more than $15 \mathrm{~s}$, they will experience slope illusion [5]. Due to misjudgment of a slope, a downhill section of a back slope is perceived as an uphill section, and the driver's behavior of changing gears and accelerating leads to an increased occurrence of traffic accidents. At present, the remediation measures for slope illusion mainly include adding guardrail columns, road widening, and plant landscape setting.

Plant landscapes can visually slow down a road's slope and alleviate slope illusion when driving downhill as well as beautify the environment while reducing visual fatigue which improves the stability and safety of driving.

\section{Materials and Methods}

The road and roadside plants in this study are modeled by UC-win/road software. Through a simulation driving simulation experiment, the drivers' physiological index data are collected, the data are summarized with Excel software, and the data are then selected and processed by SPSS software. Finally, the experimental data are compared and analyzed.

2.1. Selection of Experimental Objects. Referring to the object selection of relevant experiments, it was found that the sample number of driving simulation experiments was between 12 and 40 people [6]. A total of 20 people with driving experience were selected in the experiment, including 15 men and 5 women, aged between 20 and 50, with more than 3 years of driving experience. All subjects had good visual acuity, no color blindness, and normal hearing.

2.2. Road Alignment Design. The experimental section selected was a two-way four lane road with a single lane width of $3.75 \mathrm{~m}$ and a central isolation bandwidth of $2 \mathrm{~m}$. The total length of the road is $2.5 \mathrm{~km}$, of which $0 \sim 1000 \mathrm{~m}$ is the downhill section with a gradient of $-4 \%, 1000 \mathrm{~m} \sim 1500 \mathrm{~m}$ is the downhill section with a gradient of $-1 \%$, and $1500 \sim 2500 \mathrm{~m}$ is the downhill section with a gradient of $-4 \%$. The section of $0 \sim 1500 \mathrm{~m}$ was used as the test section, as shown in Figure 1.

2.3. Selection of Road Landscape Factors. Although the specification has relevant provisions on the height, spacing, roadside distance, and color of road landscape, there is no quantitative comparison and in-depth research on the impact of different road landscape factors on drivers' driving behavior, mental state, and attention distribution. So, this experiment studied the following four landscape factors that may affect driving behavior: (1) height, (2) spacing, (3) roadside distance, and (4) color. These factors involve all aspects of road landscape design, and each factor corresponds to three levels of which level 1 is the appropriate value, and levels 2 and 3 are the extreme values. Referring to the relevant code provisions for planning and design of urban road greening [7], the plant spacing of street trees shall be subject to the crown width of their tree species in their prime, and the minimum planting plant spacing shall be $4 \mathrm{~m}$. The minimum distance from the trunk center of the street tree to the outside of the curb shall be $0.75 \mathrm{~m}$.

The road landscape setting of the road section in the experimental group studying single factors only changes the level of the factors studied in this group. For the factors not studied in this group, the moderate value of this factor is adopted. The selection of the plant factor level is shown in Table 1 (appropriate: different from the background color; only green: the same as the background color).

2.4. Road Landscape Design. The section with moderate values for all factors is taken as the reference section. The experimental scene is shown in Figure 2.

2.5. Experimental Procedure. The specific experimental process is as follows:

(1) The subjects arrived at the traffic safety simulation laboratory before the formal start of the experiment and were briefly introduced to the driving machine and operating procedures.

(2) The subjects drove and conducted practical operations in the driving simulator for 2 minutes to reduce the excitement or tension of using the simulator for the first time.

(3) The drivers were informed of the driving route in advance and allowed to drive freely according to their driving habits. To avoid the subject's driving behavior from being affected by other subjective or objective factors, the scene was set to a highly idealized state. No other vehicles were set up on the road in the same direction as the subject, and the speed of the subject did not exceed $100 \mathrm{~km} / \mathrm{h}$ when driving. 


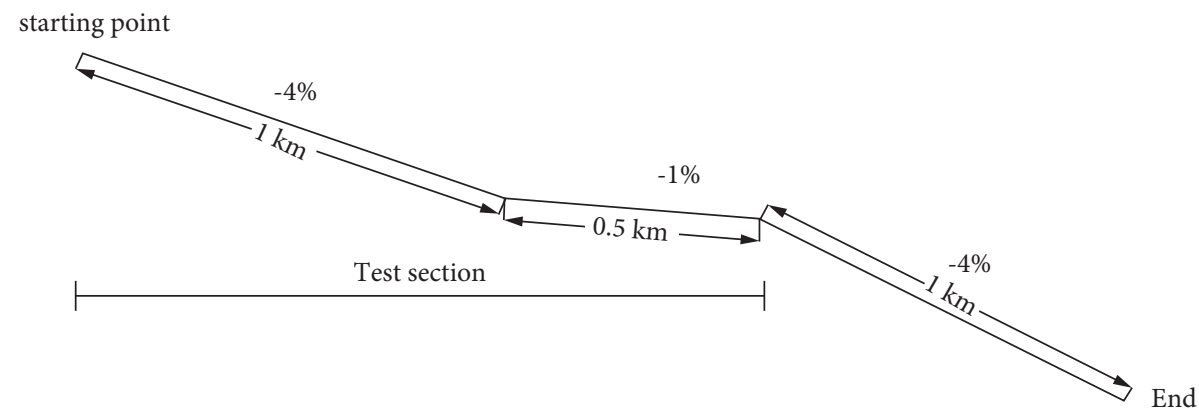

FIGURE 1: Experimental road alignment.

TABLE 1: Values of factor level.

\begin{tabular}{lcccc}
\hline Level & $\begin{array}{c}\text { Plant spacing } \\
(\mathrm{m})\end{array}$ & $\begin{array}{c}\text { Height } \\
(\mathrm{m})\end{array}$ & $\begin{array}{c}\text { Roadside } \\
\text { distance }\end{array}$ & Color \\
\hline Level 1 & 10 & 3 & 0.75 & Appropriate \\
Level 2 & 4 & 1.5 & 0.5 & Only green \\
Level 3 & 20 & 6 & 1.5 & Colorful \\
\hline
\end{tabular}

(4) During the driving process, the subjects were verbally asked one open question by the experimenter in each scene to investigate the driver's feelings about the current road section landscape.

(5) After completing the driving task of each scene, let each subject rest for $2 \mathrm{~min}$, so that the mental state of the subjects can be consistent with the previous road section, and try to reduce the excessive experimental error caused by the driver's own problems.

2.6. Experimental Data Collection. The driving simulation system in the laboratory itself provides an interface for the final export of vehicle data, including analog input and output data (DIO/AIO), vehicle speed, lateral position offset, and other parameters. According to the needs of the experiment, select the simulation system data to be exported and then click log output to export the data, as shown in Figure 3.

\section{Analysis of Landscape Factors on Driving Behavior Indicators}

3.1. Processing of Experimental Data. In the whole simulation test, the unnecessary data are selectively eliminated according to the state change of the vehicle in the line and whether the data are abnormal. The exported data format is processed and classified by Excel, and the data to be analyzed are retained. Finally, the selected data include time, speed, and lateral position offset. After processing, it is saved as an. XLS file, and the SPSS software is used to select and process the data to calculate the mean value of velocity and lateral position offset in each scene. The mean value is the central tendency of numerical data, and its calculation formula is as follows:

$$
\bar{X}=\frac{X_{1}+X_{2}+\cdots+X_{n}}{n}=\frac{\sum_{j=1}^{n} X_{j}}{n} .
$$

\subsection{Relationship between Landscape Factors and Driving} Behavior Indicators. To study the impact of various levels of selected landscape factors on drivers' experience and driving behavior, mathematical analysis methods were used to analyze driving behavior indicators. The mean value of speed and the mean value of lateral position deviation were selected as the characteristic indicators. Speed is a commonly used indicator for evaluating driving behavior, and it can reflect the driving state of the driver on a section of road. The change in the lateral position displacement parameter reflects the driver's ability to control the vehicle laterally. The greater the displacement of the vehicle's lateral position recorded in the driving simulator, the more unstable the vehicle's travel and the weaker the driver's control over the vehicle. In the whole simulation test, the unnecessary data are selectively eliminated according to the state change of the vehicle in the line and whether the data are abnormal. The exported data format is processed and classified by Excel, and the data to be analyzed are retained. Finally, the selected data include time, speed, and lateral position offset. After processing, it is saved as an. XLS file, and the SPSS software is used to select and process the data to calculate the mean value of velocity and lateral position offset in each scene. The following are the experimental results obtained under different factor levels.

The analysis results under the plant spacing factor are shown in Table 2 . The average speed is the largest at a plant spacing of $20 \mathrm{~m}$ and the smallest at a plant spacing of $4 \mathrm{~m}$; the horizontal position average is the largest at a plant spacing of $20 \mathrm{~m}$ and at a plant spacing of $10 \mathrm{~m}$, which is the smallest under this level. Most subjects believed that the ability to perceive slope was level $2>$ level $1>$ level 3 , indicating that the plant spacing was too large, which is not conducive to the driver's judgment of slope. If the plant spacing at level 2 is too small, it will bring a slight dazzling feeling to the driver, and the driver will choose to reduce the speed due to the fear of not being able to respond to emergencies in time. If the distance between level 3 was too large, the perception of speed was reduced, so the average speed was larger than that of level 1 and 


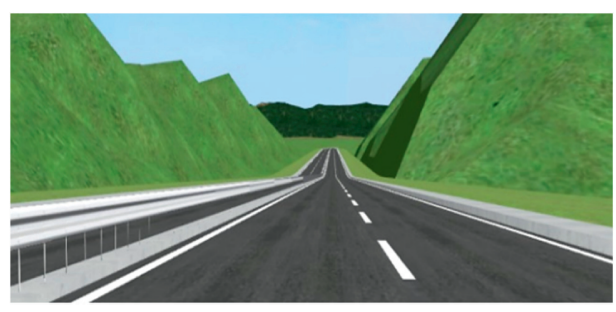

(a)

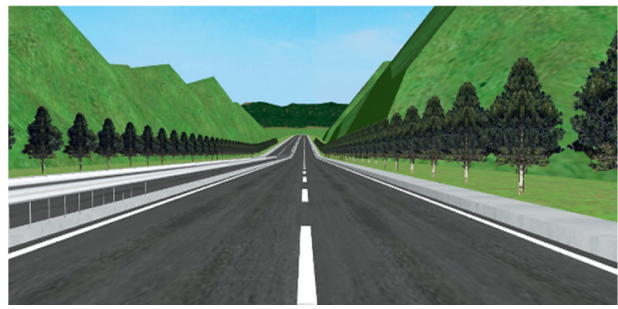

(c)

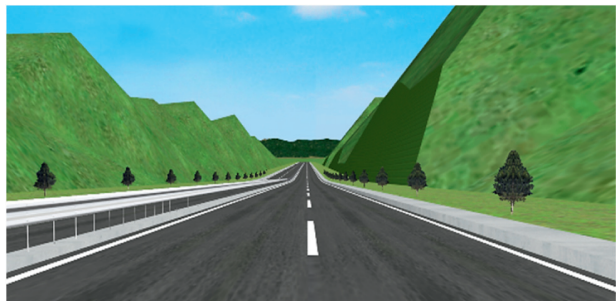

(e)

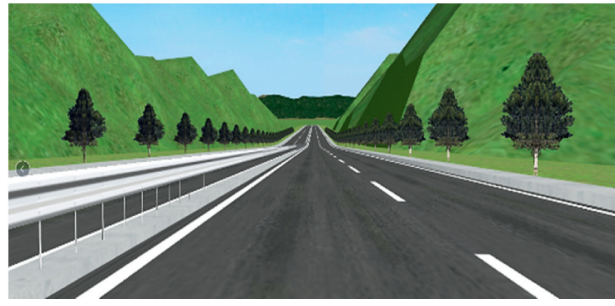

(g)

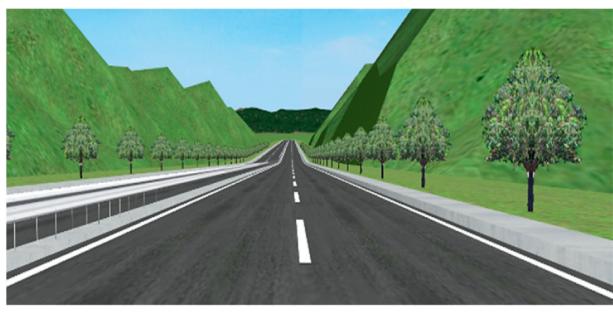

(i)

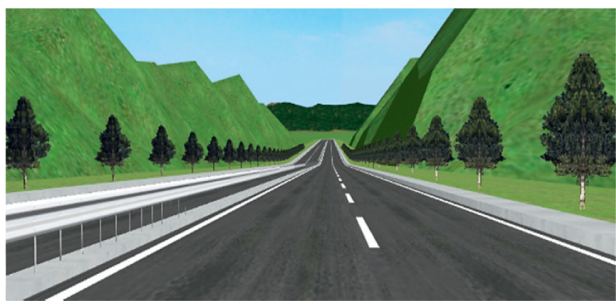

(b)

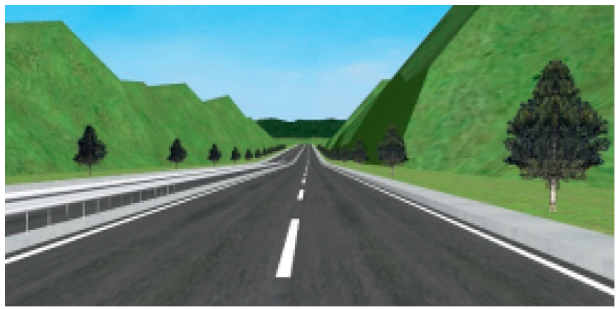

(d)

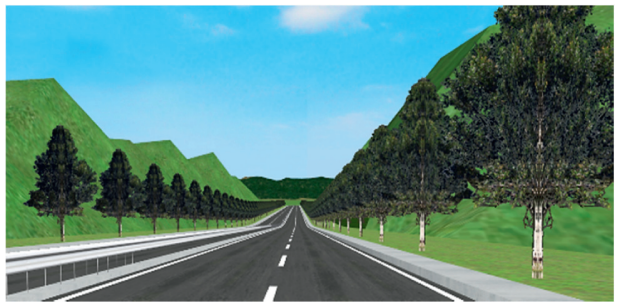

(f)

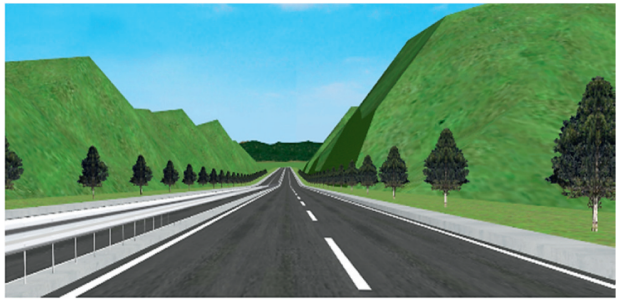

(h)

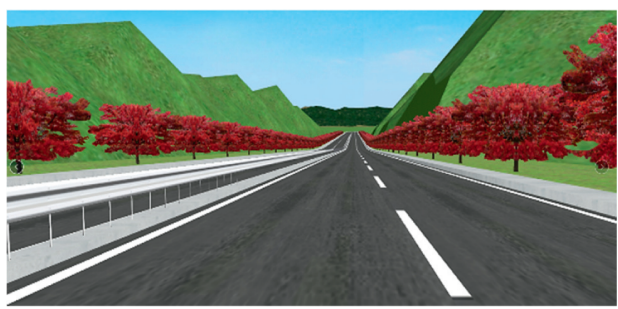

(j)

Figure 2: Scene map corresponding to each landscape factor. (a) Not processed. (b) Reference road section scene. (c) Plant spacing factor level. (d) Plant spacing factor level 3. (e) Height factor level 2. (f) Height factor level 3. (g) Roadside distance factor level 2. (h) Roadside distance factor level 3. (i) Color factor level 2. (j) Color factor level 3.

level 2. To reduce the dazzling feeling, the driver naturally drives in the center, so the average value of the horizontal position of level 2 was too large. Excessive plant spacing in level 3 appeared to have a wide field of vision, which caused the driver's mental state to relax fatigue. Therefore, the average value of the horizontal position of level 3 was greater than that of level 1 and level 2 .

The analysis results under the height factor are shown in Table 3. The average speed of level 2 was much larger than that of level 1 and level 3; the average value of the lateral position was the largest at a height of $1.5 \mathrm{~m}$ and the smallest at a height of $3 \mathrm{~m}$. Through the inquiries to the driver, it can be seen that most of the subjects believed that the level of perception of the slope was level $1>$ level $2>$ level 3 . The height of plants was too low or too high, which was not in alignment with the driver's perception and judgment of the slope. A lower height will make the driver's field of vision wider and feel comfortable when driving, but this feeling will 


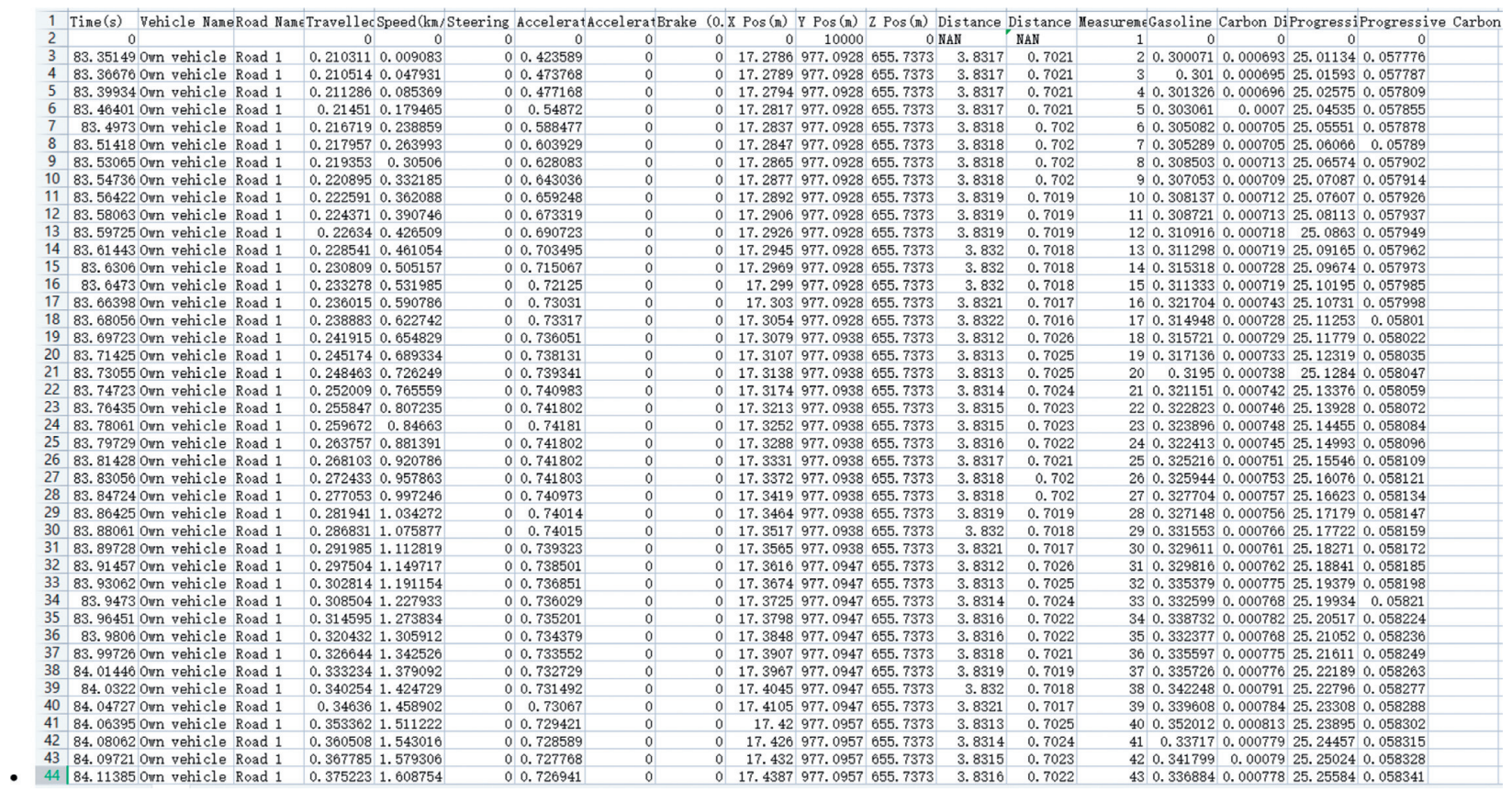

FIgURe 3: Export data.

TABLe 2: Driving behavior index results of various levels of plant distance factors.

\begin{tabular}{lccc}
\hline \multirow{2}{*}{ Driving behavior index } & \multicolumn{3}{c}{ Plant spacing (m) (level) } \\
& $1: 10 \mathrm{~m}$ & $2: 4 \mathrm{~m}$ & $3: 20 \mathrm{~m}$ \\
\hline Mean speed $\left(\mathrm{km} \cdot \mathrm{h}^{-1}\right)$ & 74.87 & 72.13 & 88.43 \\
Mean lateral position $(\mathrm{m})$ & 1.57 & 2.21 & 2.47 \\
\hline
\end{tabular}

TABle 3: Driving behavior index results under various levels of height factor.

\begin{tabular}{lccc}
\hline \multirow{2}{*}{ Driving behavior index } & \multicolumn{3}{c}{ Height (m) (level) } \\
& $1: 3 \mathrm{~m}$ & $2: 1.5 \mathrm{~m}$ & $3: 6 \mathrm{~m}$ \\
\hline Mean speed $\left(\mathrm{km} \cdot \mathrm{h}^{-1}\right)$ & 80.3 & 92.3 & 75.94 \\
Mean lateral position $(\mathrm{m})$ & 2.03 & 2.21 & 2.36 \\
\hline
\end{tabular}

cause the driver to reduce the speed control, and the lateral position offset is larger, so the average speed and lateral position of level 2 are both too large. Excessive height will give the driver a sense of oppression, and at the same time, plants that are too high may obstruct the driver's vision. The driver will drive closer to the center of the road to reduce oppression and avoid blind spots in the field of vision. Therefore, the offset of the horizontal position will be large, which is not conducive to the stable driving of the vehicle downhill.

The analysis results of the roadside distance factor are shown in Table 4. The roadside distance factor level 3 has the largest average speed, and level 2 has the smallest average speed; level 1 has the smallest horizontal position average value, indicating that the roadside distance factor level 1 is better than other levels. As for the perception of slope, it was found through verbal inquiry that there was no significant
TABLE 4: Results of driving behavior indicators under various levels of roadside factors.

\begin{tabular}{lccc}
\hline \multirow{2}{*}{ Driving behavior index } & \multicolumn{3}{c}{ Curb margin $(\mathrm{m})$ (level) } \\
& $1: 0.75 \mathrm{~m}$ & $2: 0.5 \mathrm{~m}$ & $3: 1.5 \mathrm{~m}$ \\
\hline Mean speed $\left(\mathrm{km} \cdot \mathrm{h}^{-1}\right)$ & 78.43 & 74.87 & 82.21 \\
Mean lateral position $(\mathrm{m})$ & 1.67 & 1.96 & 2.23 \\
\hline
\end{tabular}

TABle 5: Driving behavior index results under various levels of color factors.

\begin{tabular}{lccc}
\hline Driving behavior index & $\begin{array}{c}\text { Color (level) } \\
\text { appropriate }\end{array}$ & $\begin{array}{c}2 \text { : green } \\
\text { only }\end{array}$ & $\begin{array}{c}3: \\
\text { bright }\end{array}$ \\
\hline $\begin{array}{l}\text { Mean speed }\left(\mathrm{km} \cdot \mathrm{h}^{-1}\right) \\
\begin{array}{l}\text { Mean lateral position } \\
(\mathrm{m})\end{array}\end{array}$ & 86.82 & 90.34 & 81.90 \\
\hline
\end{tabular}

difference between level 1 , level 2, and level 3. The roadside distance factor has little influence on the driver's perception of slope. At roadside factor level 2, the plants are closer to the road, and the driver tends to reduce the speed and drive closer to the center of the road to alleviate the dazzling feeling caused by the plants. A large roadside distance may cause the landscape effect to be too weak and reduce the driver's perception of speed so that the speed control and steering wheel control are more random, which makes the driving speed and position deviation fluctuate greatly.

The analysis results under the color factor are shown in Table 5. Color factor level 2 has the largest average velocity, the lateral position offset is also larger, the level 3 velocity average is the smallest, and the lateral position offset has the largest average. Most subjects believed that the colors of level 
TABLE 6: Comparative analysis of subjective equivalent speed.

\begin{tabular}{lccccccccc}
\hline & Scene 1 & Scene 2 & Scene 3 & Scene 4 & Scene 5 & Scene 6 & Scene 7 & Scene 8 & Scene 9 \\
\hline SSES $(\mathrm{km} / \mathrm{h})$ & 64.41 & 75.54 & 70.29 & 68.61 & 76.54 & 66.32 & 78.54 & 80.71 & 79.42 \\
Speed illusion & +25.59 & +14.46 & +19.71 & +21.39 & +13.46 & +23.68 & +11.46 & +9.29 & +10.58 \\
Speed illusion degree (\%) & +28.43 & +15.71 & +21.90 & +23.76 & +14.96 & +26.31 & +12.73 & +10.32 & +11.75 \\
\hline
\end{tabular}

Note. Subjective equivalent speed (SSES) refers to the actual vehicle speed of the comparison scene when the perceived speed of the comparison scene is equal to the standard scene; speed illusion $=$ actual vehicle speed in the standard scene-SSES; speed illusion degree $=($ the actual vehicle speed in the standard scene-SSES)/the actual speed of the standard scene.

1 , level 2, and level 3 can well perceive the difference in slope during driving, and the ability to perceive slope is level $1>$ level $3>$ level 2. In level 3, because the colors are too bright, the driver will feel dizzy during driving, causing eye fatigue, which was not conducive to safe driving. Therefore, the dizziness can be reduced by reducing the speed and deviating from the roadside landscape, making driving obtain a larger position shift. The color of plants in level 2 was only green. This color can give the driver a comfortable feeling, but this color was similar to the color of the surrounding environment. Over time, driving in this environment will produce monotony and fatigue, which increases the driving speed and position deviation. The color of the plants in level 1 was greenish but is slightly different from the surrounding environment. This color can form a significant color difference with the surrounding environment, which will neither bring a feeling of dizziness nor cause the driver to experience long-term driving burnout.

In summary, through the data analysis of the driving behavior indicators of the experimenters, it is found that there are significant differences in the impact of the plant spacing, height, roadside distance, and color on the driver at various levels.

\section{Comparison of the Results}

In the above analysis of a single factor, while changing a single variable and finding that distinct factors have different degrees of impact on the driver, it was concluded that the following was an excellent combination: scene height of $3 \mathrm{~m}$, spacing of $10 \mathrm{~m}$, roadside distance of $0.75 \mathrm{~m}$, and an appropriately matched color. However, the purpose of the experiment was to improve the illusion of slope and better control vehicle speed by setting the plant landscape, so the effect needs to be compared with an unprocessed scene. The speed illusion and degree of illusion produced by each scene are compared by measuring the subjective equivalent speed, as shown in Table 6.

It can be seen from the table that in these 9 scenes, there was an illusion of speed and a certain degree of speed overestimation, which show that setting a plant landscape can effectively enhance the perception of speed. The subjective equivalent speed in scenario 1 is $64.41 \mathrm{~km} / \mathrm{h}$ (corresponding to a perceived vehicle speed of $90 \mathrm{~km} / \mathrm{h}$ ), and the speed overestimation is $28.43 \%$. Comparing scenario 1 with other scenarios, the speed was overestimated, while in related scenarios, the overestimation of vehicle speed is less than $28.43 \%$. Therefore, setting a landscape with a height of $3 \mathrm{~m}$, an interval of $10 \mathrm{~m}$, a roadside distance of $0.75 \mathrm{~m}$, and an appropriate color match on the downhill section will help a driver maintain a safe driving speed.

\section{Conclusion}

The influence of different plant landscape factors on the slope illusion, speed illusion, and stability produced by the driver is quantitatively analyzed, and the research conclusions are as follows:

(1) By comparing the experimental data of each scene, it is found that different levels of plant landscape plant spacing, height, roadside distance, and color factors will affect drivers to varying degrees. There are significant differences in driving behavior indicators such as speed and lateral position deviation of drivers. Only by arranging appropriate plant spacing, height, roadside distance, and color can the adverse impact on a driver be reduced, and a driver can have a comfortable feeling when driving to maintain vehicle stability and safety.

(2) Through the oral inquiry of the experimenter, it is found that a driver's ability to perceive the slope of scene 1 is greater than that of other scenes; that is, a driver is very good in a landscape with a height of $3 \mathrm{~m}$, a spacing of $10 \mathrm{~m}$, a roadside distance of $0.75 \mathrm{~m}$, and an appropriate color match. It can reduce the illusion of the slope caused by the influence of the slope difference and slope length.

(3) By measuring the subjective equivalent velocity in each scene, it is found that setting up a plant landscape can effectively improve drivers' speed perception ability. There are varying degrees of speed overestimation in 9 scenes. The overestimation of the speed during the downhill process is helpful to avoid unintentional driver acceleration and to maintain a steady speed. Among them, scenario 1 has the strongest speed perception ability of the driver and the highest degree of speed overestimation. Therefore, a height of $3 \mathrm{~m}$, spacing of $10 \mathrm{~m}$, roadside spacing of $0.75 \mathrm{~m}$, and appropriate color matching can be set to allow a driver to better control the speed of the vehicle and ensure driving safety when driving downhill.

\section{Data Availability}

The data used to support the findings of this study are included within the supplementary information files. 


\section{Conflicts of Interest}

The authors declare that there are no conflicts of interest regarding the publication of this paper.

\section{Supplementary Materials}

This section includes research data. (Supplementary Materials)

\section{References}

[1] G. Yuan and J. Cheng, "Illusion and its application in highway alignment design," China Journal of Highway and Transport, vol. 33, pp. 19-22, 2002.

[2] Y. Lu, Highway Horizontal and Vertical Design Based on Driver's Visual Characteristics, Chang'an University, Xi'an, China, 2013.

[3] Q. Guo, G. Wu, and F. Wang, "Simulation analysis of illusion animation caused by road alignment and roadside environment," Journal of Chongqing Jianzhu University: Natural Science Edition, vol. 25, no. 5, pp. 40-44, 2006.

[4] G. Qi, Research on the Formation Mechanism of Road Slope Illusion and Improvement Measures, Chongqing Jiaotong University, Chongqing, China, 2017.

[5] Q. Guo, Analysis of the Illusion Caused by the Road Alignment and Roadside Environment and the Research on Governance Measures, Master's thesis, Chongqing Jiaotong University, Chongqing, China, 2006.

[6] T. Xu, Y. Wu, and Z. Ma, "Determination of the optimal setting length of road landscape," Journal of Chang'an University, vol. 33, no. 2, Xi'an, China, 2013.

[7] China Construction Industry Press, Urban Road Greening Planning and Design Code, China Construction Industry Press, Beijing, China, Cjj75, 1997. 